\title{
The challenge of treating complex knee instability
}

\author{
V. Musahl ${ }^{1} \cdot$ S. Zaffagnini ${ }^{1} \cdot$ R. LaPrade $^{1} \cdot$ M. T. Hirschmann ${ }^{1} \cdot$ J. Karlsson $^{1}$
}

Received: 25 March 2015 / Accepted: 27 May 2015 / Published online: 3 June 2015

(C) European Society of Sports Traumatology, Knee Surgery, Arthroscopy (ESSKA) 2015

As clinicians it is humbling to accept the challenge of treating patients with complex knee instability. Not only is our pre-operative evaluation sometimes limited and complex surgical techniques less standardized, there are also a multitude of surgical and post-surgical complications that are waiting for us and many revision operations to be done. In this special theme issue we chose a strategy of deconstructing the topic of "complex knee instability" into multiple smaller, and easier to digest, subtopics: anteromedial, anterolateral, posteromedial and posterolateral corner ligament pathology and associated bony alignment and meniscal issues which are a necessary part of the workup of complex knee injuries. In the absence of Level I evidence for treatment of multiple ligament injuries, as clinicians we must be aware of knee instability patterns and their combinations, all while not forgetting the neurovasculature, whose evaluation and treatment comes first and often necessitates benign neglect of the multiple ligament injury.

As we reflect on our own clinical practices, we often make the mistake of assuming that a case is an "isolated injury", when in fact an isolated knee ligament injury is extremely rare, if not inexistent. Taking the anterior cruciate ligament (ACL) for example, when was the last time you treated an ACL tear without bone bruises, without MRI evidence of "oedema" or "high signal intensity" in the capsular structures and absolutely no injury to the articular cartilage or menisci? It depends on your point of view, but "looking for" concomitant injury sometimes affords additional pre-operative workup, portals or approaches.

V. Musahl

vmusahl@gmail.com

1 University of Pittsburgh Medical Center, Pittsburgh, PA, USA
It is important to recognize that the workup of revision complex knee cases requires more than the physical examination to help decipher the cause of the original failed reconstruction and to ultimately provide the best outcome for the patient. The patient's alignment, sagittal plane slope, status of the menisci and articular cartilage surfaces and associated concurrent ligament injuries must all be assessed for every patient and for every case.

Take for example the recently described "hidden meniscus lesion" [7]. To quote Jack Hughston from almost 50 years ago, you may not have seen it in the past, but how many times has it seen you? In some cases, this lesion is not only a tear of the meniscus periphery but also deficiency of the entire posteromedial corner. If missed, high stresses will be exerted on the ACL graft during pivoting manoeuvres, eventually leading to failure of ACL reconstruction. Similarly, meniscal root tears are often missed and the effects of missing these tears can affect the outcomes of associated ligament reconstructions and the development of osteoarthritis. Prospective studies have noted that these tears are often associated with ACL tears and other complex knee injuries [4]. Thus, it is our obligation to assess for these issues pre-operatively on MRI scans and to probe the meniscal root attachments and posteromedial and posterolateral meniscocapsular attachments in every case.

While optimal surgical timing of repairs and reconstruction is poorly defined, this special theme issue will also focus on knee anatomy and anatomical reconstructions. Repair of avulsed ligaments and tendons, whether soft tissue or bony avulsions, can successfully be performed in the acute phase; with the understanding that acute surgery for complex knee ligament injuries is associated with an increased risk of arthrofibrosis [6]. However, tendon avulsions may be irreparable in the chronic setting necessitating allograft reconstruction with less desirable outcomes. There 
is an absolute lack of high-level evidence supporting surgical timing of complex knee ligament surgery, warranting future research. Several articles in this special theme issue will discuss surgical techniques and report on the clinical outcomes of complex knee meniscus repairs and ligament repairs and reconstructions.

The articles in this special theme issue provide the reader with anatomical and biomechanical insights into the peripheral structures of the knee that have been better understood in these recent years. Peripheral structures are important in guaranteeing a decreased risk of failure and maintenance of a good functional outcome over time.

In this special theme issue you will find the historical information on the first attempt to address these lesions as well as long-term results of combined reconstructions performed by pioneer surgeons who, more than 25 years ago, believed that it was important to address all lesions causing instability and not to focus only on treating the central pivot. Our mission is to improve clinical outcomes for patients. Therefore, we must develop instruments that can adequately assess the global laxity of a joint in all directions: anterior-posterior, varus-valgus, and rotatory. Zaffagnini et al. [8] have reported in several studies that global knee laxity can be assessed pre-operatively under anaesthesia, which can be used to guide the surgeon to a particular injury pattern. Furthermore, using non-invasive devices, global knee laxity can also be assessed post-operatively, and for the first time can provide quantitative and objective outcome data on knee laxity [3,5]. In addition, the use of stress radiographs is recommended to help decipher which ligaments are torn. This will enable objective measurements of surgical outcomes that can be obtained uniformly and across multiple centres [1,2].

In this issue we compiled the latest information on this topic that due to its complexity is often not addressed in the literature, while we have tried to enlighten the importance of diagnosis and treatment of these lesions.

We hope you will enjoy this special theme issue of Knee Surgery Sports Traumatology and Arthroscopy.

\section{References}

1. Dejour D, Ntagiopoulos PG, Saggin PR, Panisset JC (2013) The diagnostic value of clinical tests, magnetic resonance imaging, and instrumented laxity in the differentiation of complete versus partial anterior cruciate ligament tears. Arthroscopy 29(3):491-499

2. Jung TM, Reinhardt C, Scheffler SU, Weiler A (2006) Stress radiography to measure posterior cruciate ligament insufficiency: a comparison of five different techniques. Knee Surg Sports Traumatol Arthrosc 14(11):1116-1121

3. Hoshino Y, Araujo P, Ahlden M, Samuelsson K, Muller B, Hofbauer M, Wolf MR, Irrgang JJ, Fu FH, Musahl V (2013) Quantitative evaluation of the pivot shift by image analysis using the iPad. Knee Surg Sports Traumatol Arthrosc 21(4):975-980

4. LaPrade RF, Ho CP, James E, Crespo B, LaPrade CM, Matheny LM (2015) Diagnostic accuracy of $3.0 \mathrm{~T}$ magnetic resonance imaging for the detection of meniscus posterior root pathology. Knee Surg Sports Traumatol Arthrosc 23(1):152-157

5. Lopomo N, Signorelli C, Bonanzinga T, Marcheggiani Muccioli GM, Neri MP, Visani A, Marcacci M, Zaffagnini S (2014) Can rotatory knee laxity be predicted in isolated anterior cruciate ligament surgery? Int Orthop 38(6):1167-1172

6. Mook WR, Miller MD, Diduch DR, Hertel J, Boachie-Adjei Y, Hart JM (2009) Multiple-ligament knee injuries: a systematic review of the timing of operative intervention and postoperative rehabilitation. J Bone Joint Surg Am 91(12):2946-2957

7. Sonnery-Cottet B, Conteduca J, Thaunat M, Gunepin FX, Seil R (2014) Hidden lesions of the posterior horn of the medial meniscus: a systematic arthroscopic exploration of the concealed portion of the knee. Am J Sports Med 42(4):921-926

8. Zaffagnini S, Signorelli C, Lopomo N, Bonanzinga T, Marcheggiani Muccioli GM, Bignozzi S, Visani A, Marcacci M (2012) Anatomic double-bundle and over-the-top single-bundle with additional extra-articular tenodesis: an in vivo quantitative assessment of knee laxity in two different ACL reconstructions. Knee Surg Sports Traumatol Arthrosc 20(1):153-159 